\title{
Spectral Observations of the Collimated Outflows in LkH $\alpha 225$ and GM1-27
}

\author{
T. Yu. Magakian and T. A. Movsessian \\ Byurakan Astrophysical Observatory, National Academy of Sciences, \\ Armenia
}

\section{LkH $\alpha 225$}

The young emission-line star LkH $\alpha 225$ (V1318 Cyg) with a tiny cometary nebula is located in the small group of very young stars around the well-known Herbig Ae/Be star BD $+40^{\circ} 4124$. LkH $\alpha 225$ was discovered by Herbig (1960) and studied by several authors (see for references Ibragimov et al.,1988). The star undergoes strong variations of its light (nearly 3 magn.) in visible light as well as in infrared. Spectral type of LkH $\alpha 225$ is very uncertain, but its high luminosity $\left(L_{b o l}=460\right.$ $\left.L_{\odot}\right)$ and mass $\left(5.5 M_{\odot}\right)$, as well as the presence of nebulosity suggests that it also belongs to the Herbig Ae/Be class of pre-main sequence (PMS) stars. This small and perhaps variable nebulous appendage on the $\mathrm{NW}$-side of the star can be readily seen on the plates obtained with the 2.6-meter telescope of Byurakan Observatory.

LkH $\alpha 225$ was observed on 1992 March 26 with the TIGER multi-pupil fiberless spectrograph at the prime focus of the 6-meter telescope of Special Astrophysical Observatory (Zelenchuk, Russia). A matrix of $9 \times 10$ spectra was recorded in time-resolved mode. Each spectrum corresponds to $0.7 \times 0.7$ arcsec in the sky and has a linear reciprocal dispersion of about $2 \mathrm{~A} /$ pix. Red spectral range was observed. The spatial and spectral resolution was improved by the special computer treatment after the observations, and then subsequent reductions of spectra were performed.

On the restored images we can see the narrow zone of [SII] and [OI] emission on the background of reflected continuum and $\mathrm{H} \alpha$-emission. This emission is elongated in the direction of the cometary nebula. We concluded to the presence of a collimated outflow, originating in $\mathrm{LkH} \alpha 225$. The length of the studied part of the outflow is about 3 arcsec. Its probable total length could be 6-7 arcsec. The outflow could be discerned from background emission also by its negative radial velocity (about -120 and $+80 \mathrm{~km} \mathrm{~s}^{-1}$ in the outflow and background respectively. Strong variations of electron density along the jet can also be seen.

\section{GM1-27}

The cometary nebula GM1-27, of classic conical shape, included in the list of Gyulbudaghian \& Magakian (1977), was first identified on the charts of the Palomar Observatory Sky Survey. On deep images, a faint star $(I=17)$ was found near the apex of the cometary nebula. Further polarimetric observations confirmed that the nebula is illuminated by this single star (Neckel \& Staude, 1987). 
Spectral investigations of GM1-27 were carried out by Magakian (1988) and Neckel \& Staude (1987). From indirect spectral evidences, the existence of directed outflow was inferred. By means of CCD imagery through an [SII] filter, Neckel \& Staude (1987) found an emission knot, located westward from the star, against the reflected background. This they interpreted as a Herbig-Haro (HH) object inside the nebula.

New observations were performed on 14/15 October 1991 at the prime focus of the 6-meter telescope with the UAGS spectrograph and two-dimensional photoncounting system. Spectra were obtained with effective spectral resolution of about $3 \mathrm{~A}$, in the $6200-6900$ and $4500-5200 \mathrm{~A}$ spectral ranges. The spatial resolution was $0.35 \mathrm{arcsec} / \mathrm{pixel}$. The slit was oriented along the axis of the cometary nebula.

An emission knot 4.5 arcsec in length is superimposed on the reflected continuous spectrum of the cometary nebula. In the observed spectral range it shows $\mathrm{H} \alpha, \mathrm{H} \beta,[\mathrm{OIII}],[\mathrm{OI}]$ and [SII] emission lines, typical of $\mathrm{HH}$ objects. Isointensity maps of $\mathrm{H} \alpha$, [NII], [OIII], [OI] and [SII] emission lines were produced. The most remarkable feature of these maps is the inclination of certain emission lines, so that their radial velocity increases with distance from the driving star. This effect can be readily seen for the low-excitation [OI] and [SII] lines, but it weakens for [NII] and virtually does not exist for $\mathrm{H} \alpha$ and [OIII]. We assume that the star in GM1-27 belongs to Herbig Ae/Be star type. The emission knot in GM1-27 is a high-velocity outburst, directed along the axis of the cometary nebula. The data concerning the radial velocities and the spatial distribution of the emission lines, clearly indicate the presence of stratification and of two regions of shock excitation. Somewhat similar effects were already observed in some HH objects, for example, in HH34 (Bührke et al., 1988) and in GGD34 (Gomez de Castro et al.,1993). In the case of GM1-27 the regions of [SII] and [OIII] emission overlap, but this could be the result of projection, and in fact both zones could be spatially separated. We identify these zones as the bow-shock and the Mach disk. In fact, the inclination of low-excitation lines can be considered as the evidence that we are resolving not only the regions of bow-shock and Mach disk (with the separation between them about $10^{17} \mathrm{~cm}$ ), but also the bow-shock itself.

Acknowledgments. This work was partly supported by grant A-02-044 of the ESO C\&EE Astronomy Programme, as well as by grants from the Armenian Professional Society (T.Yu.M.) and the International Science Foundation (T.A.M.)

\section{References}

Bührke, T., Mundt, R. \& Ray, T. P. 1988, A\&A, 200, 99

Gomez de Castro, A. I., Miranda, L.F. \& Eiroa, C. 1993, A\&A, 267, 559

Gyulbudaghian, A. L. \& Magakian, T. Yu. 1977, Pis'ma v AZh 3, 113

Herbig, G. H. 1960, ApJS, 4, 337

Ibragimov, M. A., Mel'nikov, S. Yu., Chernyshov, A.K. \& Shevchenko, V.S. 1988, Afz, 29, 633

Magakian, T. Yu. 1988, Afz, 28, 599

Neckel, Th. \& Staude, H. J. 1987, ApJ, 320, L145 